\title{
Single Nucleotide Polymorphism to Disclose Severe Side-effects or Proper Dosage for Each Patient
}

Key words: azathioprine, thiopurine S-methyl transferase (TPMT), systemic lupus erythematosus, immunosuppressant

Recently, the importance of single nucleotide polymorphism (SNP) has been stressed. The discovery of the disease-related gene can disclose disease susceptible or resistant persons, such as noted in persons resistant to AIDS virus infection. Genetic polymorphism is also important in drug-responsiveness, and also in the occurrence of severe side effects in each patient.

Azathioprine is one of the most frequently used immunosuppressants in the treatment of autoimmune diseases, such as systemic lupus erythematosus, poly- and dermato-myositis, and polyarteritis nodosa. Although infrequent, we sometimes experience marked leukopenia during the treatment with azathioprine, which forced us to stop use of the drug immediately.

Azathioprine, mercaptopurine and thioguanine are innactivated by S-methylation which is catalyzed by the enzyme, thiopurine S-methyl transferase (TPMT) (1). It has recently been found that some patients whose erythrocytes show a low enzymatic activity of TPMT often show severe and potentially fatal leukopenia when they are treated with conventional doses of azathioprine or mercaptopurine.

Thiopurine S-methyltransferase activity shows codominant genetic polymorphism $(2,3)$. It was reported that about $90 \%$ of white and black persons have a high TPMT activity, and $10 \%$ have an intermediate activity caused by heterozygosity at the TPMT locus. About 1 in 300 persons inherits TPMT deficiency as an autosomal recessive trait. It has been shown that less efficient methylation of mercaptopurine and azathioprine results in more extensive conversion of these purines to active thioguanine metabolites, which causes marked suppression of bone marrow. This indicates that patients who have TPMT deficiency accumulate higher levels of thioguanine nucleotides if they receive standard doses of mecaptopurine or azathioprine.

Patients who have intermediate TPMT activity that is caused by heterozygosity at the TPMT locus can be treated with $50 \%$ doses of azathioprine, and those who have a homozygous defect in TPMT locus can prevent severe hematopoietic toxicity if the azathioprine dose is decreased 8-15 fold (4). Consequently, it is quite important to know in advance whether a patient who will be treated with thiopurine derivatives, has genetic polymorphism at TPMT site.
The PCR-based methods for detection of these TPMT mutations in the genomic DNA were shown to have excellent concordance with phenotypes. Three mutant alleles that are associated with TPMT deficiency were isolated and characterized: TPMT*2, TPMT*3A and TPMA*3C. In Caucasian populations, $89-94 \%$ of individuals have a high TPMT activity, 6$11 \%$ intermediate and $0.3 \%$ low, resulting from genetic polymorphism (5). DNA samples from 199 British Caucasian, 99 British South West Asian, and 192 Chinese individuals were analyzed for the presence of these variant alleles using polymerase chain reaction-restriction fragment length polymorphism and allele-specific polymerase chain reaction-based assays (6). The frequency of individuals with a variant thiopurine methyltransferase genotype was: Caucasians $10.1 \%$, South West Asians 2.0\%, and Chinese 4.7\%. Two TPMT*2 heterozygotes were identified in the Caucasian population, but this allele was not found in the two Asian populations. TPMT*3A was the only mutant allele found in the South West Asians (two heterozygotes). This was also the most common mutant allele in the Caucasians ( 16 heterozygotes and one homozygote) but was not found in the Chinese. All mutant alleles identified in the Chinese population were TPMT*3C (nine heterozygotes). This allele was found at a low frequency in the Caucasians (one heterozygote). African population also has this mutation (7).

The paper in this journal by Ishioka et al (8) showed that 33 (91.7\%) out of 36 Japanese rheumatic patients showed TPMT alleotype of TPMT*1/TPMT*1, while 3 (8.3\%) individuals showed TPMT*1/TPMT*3C. This mutation is same as that found in the Chinese population as described above. They also showed that all three patients with the mutant allele of TPMT*3C discontinued azathioprine treatment due to leukopenia.

When we administer a drug for patients, we know that there are good responders, poor responders and non-responders to the drug and that some patients may react adversely to it. However, we have to wait until they respond to or react adversely to the drug we have administered. As shown in this paper, we may be able to know beforehand from the information of genetic polymorphism, whether a patient will respond or show adverse effects, and may know further the proper dosage for each patient. Disease-modifying anti-rheumatic drugs (DMARDs) are effective in about 50\%, but not in the rest of the patients with rheumatoid arthritis. If we are able to know responders and non-responders of DMARDs in advance, it is not necessary to waste several months to select the right drug. 
It would become possible to anticipate the effectiveness and side effects of all drugs, not after the administration of the drugs, but in advance based on the information of genetic polymorphism as shown in the drug metabolism of azathioprine.

\section{Yoichi ICHIKAWA, MD}

St. Marianna University School of Medicine, Department of Medicine, Division of Rheumatology and Allergy, 2-16-1 Sugao, Miyamae-ku, Kawasaki 216-8512

\section{References}

1) Krynetski EY, Tai HL, Yates CR, et al. Genetic polymorphism of thiopurine s-methyltransferase: clinical importance and molecular mechanisms. Pharmacogenetics 6: 279-290, 1996.

2) Weinshilboum RM, Sladek SL. Mercaptopurine pharmacogenetics: monogenic inheritance of erythrocyte thiopurine methyltransferase activity. Am J Hum Genet 32: 651-662, 1980.
3) McLeod HL, Lin JS, Scott EP, Pui CH, Evans WE. Thiopurine methyltransferase activity in American white subjects and black subjects. Clin Pharmacol Ther 55: 15-20, 1994.

4) Krynetski EY, Schuetz JD, Galpin AJ, Pui CH, Relling MV, Evans WE. A single point mutation leading to loss of catalytic activity in human thiopurine s-methyltransferase. Proc Natl Acad Sci USA 92: 949-953, 1995.

5) Tai HL, Krynetski EY, Yates CR, et al. Thiopurine s-methylktransferase deficiency: two nucleotide transitions define the most prevalent mutant allele associated with loss of catalytic activity in Caucasians. Am J Hum Genet 58: 694-702, 1996.

6) Collie-Duguid ESR, Pritchard SC, Powrie RH, et al. The frequency and distribution of thiopurine methyltransferase alleles in Caucasian and Asian populations. Pharmacogenetics 9: 37-42, 1999.

7) Ameyaw MM, Collie-Duguid ES, Powrie RH, Ofori-Adjei D, McLeod HL. Thiopurine methyltransferase alleles in British and Ghanaian populations. Hum Mol Genet 8: 367-370, 1999.

8) Ishioka S, Hiyama K, Sato H, et al. Thiopurine methyltransferase genotype and toxicity of azathioprine in Japanese. Intern Med 38: 944-947, 1999. 